\title{
Bioprosthetic valve thrombosis following surgical aortic valve replacement: Did we all miss it?
}

\author{
Niv Ad, MD
}

\author{
From Cardiovascular and Thoracic Surgery Associates, Falls Church, Va. \\ Disclosures: Dr Ad is a consultant for Medtronic, a member of the speaker's bureau for AtriCure, proctor and \\ member of the speaker's bureau for LivaNova, on the advisory board for Nido Surgical, and co-owner for \\ Left Atrial Appendage Occlusion, LLC. \\ Received for publication July 26, 2016; accepted for publication July 26, 2016. \\ Address for reprints: Niv Ad, MD, Cardiovascular and Thoracic Surgery Associates, 2921 Telestar Court, Falls \\ Church, VA 22042 (E-mail: Niv.Ad@inova.org). \\ J Thorac Cardiovasc Surg 2016;152:981-2 \\ $0022-5223 / \$ 36.00$ \\ Copyright (c) 2016 by The American Association for Thoracic Surgery \\ http://dx.doi.org/10.1016/j.jtcvs.2016.07.064
}

The expert opinion by Egbe, Connoley, and Schaff ${ }^{1}$ is very important. It clearly indicates that the incidence and extent of bioprosthesis valve thrombosis (BPVT) following surgical aortic valve replacement (SAVR) is higher than was known. The article also sheds light on late occurrence and the potential significance of BPVT in patients with late prosthetic valve failure. Recently, another study, by Makkar and colleagues, ${ }^{2}$ raised important questions associated with this clinical phenomenon both in transcatheter aortic valve replacement (TAVR) and SAVR. In a different publication, Egbe's group from the Mayo Clinic $^{3}$ published a retrospective analysis of a large cohort representing a single-center experience and describing BPVT following surgical aortic valve replacement (AVR). In that study, BPVT was identified in 46 of 397 explanted valves $(11.5 \%)$. Sixty-five percent of those patients had their valve replacement more than a year before the reoperation and $15 \%$ underwent valve replacement more than 5 years before.

The retrospective nature of both of the aforementioned articles makes it very challenging to understand the real incidence and the mechanism of BPVT. However, the importance and immediate practical aspects of the expert opinion by Egbe and colleagues ${ }^{1}$ are the open questions directly related to the postoperative management of patients following SAVR with bioprosthesis. In many centers worldwide, most patients are not being discharged home taking any kind of anticoagulation therapy unless indicated otherwise. ${ }^{4}$ Based on the Mayo Clinic report ${ }^{3}$ and a Danish study, ${ }^{5}$ this practice should be carefully assessed in a well-controlled prospective study. The second important clinical point mentioned by Egbe and colleagues ${ }^{1}$ is the method of follow-up required. Routine echocardiography is not being performed in many centers unless clinical questions arise. ${ }^{6}$ Clear guidelines regarding echocardiography should be implemented and follow-up should be standardized. However, we should all be aware of the potential limitations of transthoracic echocardiography when

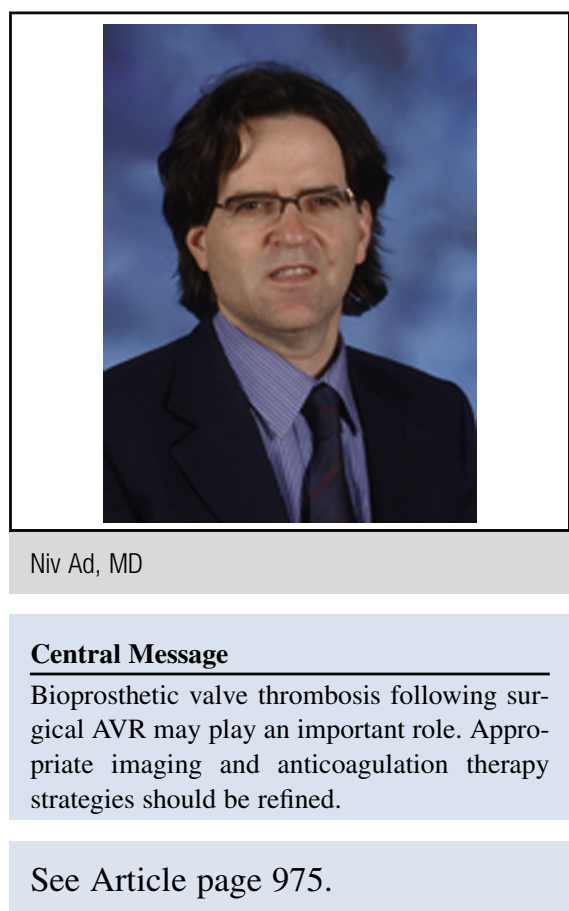

it comes to recognizing BPVT. ${ }^{2}$ Clinicians should follow signs such as an increase of $50 \%$ or transvalvular gradients since implants, abnormal leaflet mobility, and increased cusp thickness. ${ }^{2}$ In these cases, transesophageal echocardiogram or computed tomography scans with a dedicated 4-dimensional, volume-rendered computed tomography-acquisition protocol should be performed. ${ }^{2}$

Bioprosthetic valve thrombosis following AVR, whether TAVR or SAVR, is probably more prevalent and significant than was initially thought. Reports regarding this phenomenon in the surgical literature are few. ${ }^{7}$ Therefore, the detailed, retrospective study from the Mayo Clinic is very unique and important. ${ }^{3}$ Based on the current literature, valve thrombosis is more common following TAVR then SAVR, ${ }^{2}$ and probably occurs much earlier in patients undergoing TAVR, suggesting a different mechanism. Leaflet thrombosis of transcatheter valves may be explained by aggressive crimping and potential damage to bioprosthesis leaflet integrity, which may lead to early thrombosis. It is also important to recognize the potential for blood stasis at the base of the implanted valve due to the limited washout associated with the calcified native aortic valve. The final aspect that should be taken into consideration is the noncircular expansion of the transcatheter valve and the uneven stress distribution across the leaflets that may result in stress 


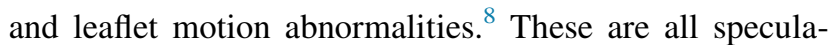
tions that require more sophisticated analysis both for leaflet integrity and flow patterns following TAVR.

The pathophysiology of BPVT following surgical AVR may be somewhat different than that for TAVR and needs to be determined using appropriate imaging and in a prospective way. The potential differences between pericardial and porcine valves are notable $\mathrm{e}^{3}$ and need to be evaluated carefully. Bioprosthesis thrombus following SAVR should also be more carefully evaluated in the context of early valve deterioration and with abnormal leaflet mobility as the precipitating factor. It is unclear how important are short events of supraventricular arrhythmias following surgery and whether postoperative rhythm monitors should be applied with a more aggressive approach to anticoagulation.

Egbe and colleagues ${ }^{1}$ provide an excellent overview that summarizes nicely the current state of evidence and future questions that need to be answered. We should all focus on understanding the mechanism and incidence of BPVT by developing the appropriate screening modalities. The result of such efforts will lead to better design of future studies to identify the patients at risk and optimize the perioperative and long-term management of patients following AVR when it comes to anticoagulation treatment. Future efforts should be focused around 3 clinical questions:

1. What is the incidence and importance of reduced leaflet motion following SAVR with bioprosthetic valves?

2. Is the reduced leaflet motion caused by thrombus formation, or is it primarily related to the valve deterioration process and the thrombus formation is secondary?
3. What are the clinical implications when it comes to imaging follow-up and anticoagulation treatment?

\section{References}

1. Egbe AC, Connolly HM, Schaff HV. Bioprosthetic valve thrombosis: What we know and what we need to know. J Thorac Cardiovasc Surg. 2016;152:975-8.

2. Makkar RR, Fontana G, Jilaihawi H, Chakravarty T, Kofoed KF, de Backer O, et al. Possible subclinical leaflet thrombosis in bioprosthetic aortic valves. $N$ Engl J Med. 2015;373:2015-24.

3. Egbe AC, Pislaru SV, Pellikka PA, Poterucha JT, Schaff HV, Maleszewski JJ, et al. Bioprosthetic valve thrombosis versus structural failure: clinical and echocardiographic predictors. J Am Coll Cardiol. 2015;66:2285-94.

4. Nishimura RA, Otto CM, Bonow RO, Carabello BA, Erwin JP III, Guyton RA, et al. 2014 AHA/ACC Guideline for the Management of Patients With Valvular Heart Disease: executive summary: a report of the American College of Cardiology/American Heart Association Task Force on Practice Guidelines. Circulation. 2014;129:2440-92.

5. Merie C, Kober L, Skov Olsen P, Andersson C, Gislason G, Skov Jensen J, et al. Association of warfarin therapy duration after bioprosthetic aortic valve replacement with risk of mortality, thromboembolic complications, and bleeding. JAMA. 2012;308:2118-25.

6. Zoghbi WA, Chambers JB, Dumesnil JG, Foster E, Gottdiener JS, Grayburn PA, et al. Recommendations for evaluation of prosthetic valves with echocardiography and Doppler ultrasound: a report From the American Society of Echocardiography's Guidelines and Standards Committee and the Task Force on Prosthetic Valves, developed in conjunction with the American College of Cardiology Cardiovascular Imaging Committee, Cardiac Imaging Committee of the American Heart Association, the European Association of Echocardiography, a registered branch of the European Society of Cardiology, the Japanese Society of Echocardiography and the Canadian Society of Echocardiography, endorsed by the American College of Cardiology Foundation, American Heart Association, European Association of Echocardiography, a registered branch of the European Society of Cardiology, the Japanese Society of Echocardiography, and Canadian Society of Echocardiography. J Am Soc Echocardiogr. 2009;22:975-1014.

7. Ohnaka M, Nishimura K, Kurokawa S. Flat fibrin thrombus deposition on tissue valve after aortic valve replacement. Ann Thorac Surg. 2010;89:2032-4.

8. Duraiswamy N, Weaver JD, Ekrami Y, Retta SM, Wu C. A parametric computational study of the impact of non-circular configurations on bioprosthetic heart valve leaflet deformations and stresses: possible implications for transcatheter heart valves. Cardiovasc Eng Technol. 2016;7:126-38. 